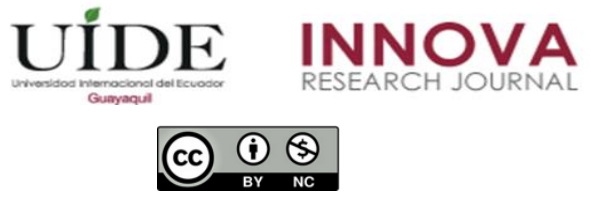

INNOVA Research Journal, ISSN 2477-9024

(Septiembre-Diciembre 2019). Vol. 4, No.3.2 pp. 40-49

DOI: https://doi.org/10.33890/innova.v4.n3.2.2019.1206

URL: http://revistas.uide.edu.ec/index.php/innova/index

Correo: innova@uide.edu.ec

\title{
Análisis de la inversión pública en educación, saneamiento y transporte en el distrito de Sanagorán, La Libertad- Perú
}

\section{Analysis of public investment in education, sanitation and transport in the district of Sanagorán, La Libertad- Peru}

Manuel Alberto Munsibay Muñoa

https://orcid.org/0000-0001-9927-5909

Lauralinda Leonor Cavero-Egúsquiza Vargas

https://orcid.org/0000-0001-5222-8865

Irma Milagros Carhuancho Mendoza

https://orcid.org/0000-0002-4060-5667

Universidad César Vallejo, Perú

Autor por correspondencia: mmunsibay@gmail.com; lauralinda.cavero@gmail.com; irmamilagros@yahoo.com

Fecha de recepción: 22 de septiembre del 2019 - Fecha de aceptación: 05 de diciembre del 2019

\section{Resumen}

La investigación tiene como objetivo analizar la inversión pública en los sectores de educación, saneamiento y transporte en Sanagorán, La Libertad - Perú. La metodología empleada para esta investigación fue de enfoque cualitativo y estudio de caso, se aplicó la técnica de la entrevista y el análisis documental. Para el análisis se reunió información de los proyectos de inversión pública y de los indicadores de cada sector en estudio, utilizando la base de datos del Banco Central de Reserva del Perú y del Ministerio de Economía y Finanzas. La investigación concluyó que más del $75 \%$ de proyectos de inversión pública se encuentran en situación de viable. En el sector educación las obras lograron beneficiar al $97.85 \%$ de la zona rural, significando el $21.24 \%$ del presupuesto para el año 2018, mientras que en saneamiento se invirtió el $24.99 \%$ enfatizando en la ampliación del sistema de agua potable por no contar con dicho servicio básico, finalmente en transporte se invirtió el $27.33 \%$ para la construcción de trochas carrózales.

Palabras Claves: inversión pública; proyectos; educación; saneamiento y transporte

\begin{abstract}
The research aims to analyze public investment in the education, sanitation and transport sectors in Sanagorán, La Libertad - Peru. The methodology used for this research was qualitative approach and case study, the technique of interviewing and documentary analysis was applied. Information was collected for the analysis of public investment projects and indicators for each sector under study, using the database of the Central Reserve Bank of Peru and the Ministry of Economy and Finance. The research concluded that more than $75 \%$ of public investment projects are viable. In the education sector, works benefited $97.85 \%$ of the rural area, which means $21.24 \%$ of the budget for 2018 , while in sanitation $24.99 \%$ was invested by emphasizing the expansion of the drinking
\end{abstract}


water system because it did not have the basic service, finally in transport was invested $27.33 \%$ for the construction of carrósal logs.

Key words: public investment; project; education; sanitation and transport

\section{Introducción}

En la actualidad la mayoría de los países en el mundo, en especial los que están en vías de desarrollo buscan financiamiento para invertir en proyectos y mejorar la calidad de vida de sus pobladores (Fondo Monetario Internacional, 2016). La Comunidad Económica Europea dentro de su plan de inversiones para el 2018 ha destinado 315,000 millones de euros y movilizar al menos 500,000 millones antes del 2020 para inversiones públicas como privadas, los objetivos fueron: a) Impulsar las inversiones; b) Aumentar la competitividad; c) Apoyar el crecimiento económico a largo plazo en la UE. Este plan fue propuesto por la Comisión Europea en el 2014, con la finalidad de subsanar los bajos niveles de inversión, el objetivo fue impulsar el crecimiento económico y el empleo. El plan de inversiones para Europa se centra en tres pilares: a) Un fondo Europeo para inversiones estratégicas: El Fondo, que fuera creado en el 2015, a través del Banco Europeo de Inversiones (BEI), viene utilizando financiamiento público para movilizar inversión privada adicional. El Fondo Europeo para Inversiones Estratégicas (FEIE) invierte en sectores muy diversos, como infraestructura, energía, investigación e innovación, banda ancha y educación. También está dispuesto a prestar apoyo a pequeñas y medianas empresas a través del Fondo Europeo de Inversiones; b) Garantizar que la financiación de la inversión llegue a la economía real, por ello se creó un Portal Europeo de Proyectos de Inversión y el Centro Europeo de Asesoramiento para la Inversión; c) Mejorar el entorno de inversión, tiene como objetivo suprimir los obstáculos, utilizando una normativa más sencilla, predecible y mejor en la UE, especialmente en los sectores de las infraestructuras, en los que las inversiones se extienden a lo largo de varios años (Comunidad Económica Europea, 2018).

En América Latina, los Sistemas de Inversión Pública han estado evolucionando, el objetivo principal fue la racionalización de los procesos de identificación, formulación, evaluación, selección, programación, ejecución y seguimiento de los proyectos y estudios básicos. En este sentido, buscan asegurar que la calidad de las inversiones, contribuyan al desarrollo y bienestar de la población, para ello se implementó marcos institucionales, normas legales, procedimientos, actividades interrelacionadas y coordinadas. Contreras, Cartes y Pacheco (2010) describen que el Sistema Nacional de Inversión Pública (SNIP) en Latinoamérica atravesó durante casi cuatro décadas un proceso de implementación gradual y ha permitido logros importantes, aunque también lamentan serios retrocesos.

En el Perú el sistema administrativo encargado de la evaluación y seguimiento de la inversión era el Sistema Nacional de Inversión Pública (SNIP), fue creado en junio del 2000. En sus inicios se atendía sólo a los proyectos de entidades del nivel nacional y regional, a partir del 2002, se incorporó a los Gobiernos Locales. El Perú se encuentra en crecimiento económicos y para reducir la brecha de las necesidades de la población se ha implementado procesos más simples de inversión, mediante el Decreto Legislativo $\mathrm{N}^{\circ} 1252$ del 01 de diciembre de 2016, vigente desde el 24 de febrero del 2017, es así que creó el Sistema Nacional de Programación Multianual y Gestión de Inversiones, que se conoce como INVIERTE.PE, sustituyendo al SNIP (Ministerio de Economía y Finanzas, 2018). 
Ministerio de economía y finanzas (2018) a través del programa multianual de inversiones del estado ha delegado a las oficinas de programación de inversiones que aprueben la viabilidad de los proyectos que son financiados sin aval del Estado, para los gobiernos regionales, locales y nacionales cuyo monto no supere los 3 millones de soles, a partir de abril del 2004 los límites se elevaron a 8 millones soles con el propósito de destinar los fondos a proyectos de los sectores de transporte y energía, para el caso de los gobiernos locales se elevó a 3 millones de soles si se realizaban con financiamiento técnico no reembolsable. Por otro lado los proyectos de inversión con aval del Estado son evaluados por la Dirección General de Programación Multianual que depende directamente del ministerio de economía y finanzas, el nuevo sistema la inversión pública está basada en las necesidades de la población con una planificación adecuada disminuyendo el tiempo de formulación y evaluación.

Por ese motivo en la provincia de Sánchez Carrión se viene desarrollando un Plan de desarrollo local concertado para el periodo 2017-2021, el mismo que incluye a ocho distritos: Huamachuco, Sartimbamba, Cochorcos, Chugay, Sanagorán, Marcabal, Sarín, Curgos y Sanagorán. Levantándose información conocida como conocimiento integral de la realidad, relativos a su sistema productivo, relacional, ambiental, poblacional y equipamental (Municipalidad de Sánchez Carrión, 2017).

Se debe resaltar que el sistema productivo de la región La Libertad dispone de una superficie agrícola de 528,763.75 hectáreas, representando el 51.72\% de superficie agrícola bajo riego/superficie agrícola total, con 53, 484.64 hectáreas representando el $19.91 \%$ que corresponde a la provincia de Sánchez Carrión, y 5,335.44 hectáreas, de los cuales solo corresponde al $9.83 \%$ al distrito de Sanagorán, que se caracteriza por la producción del ganado vacuno, ovino y cuyes, así también la ñuña, maíz, palta, lenteja, cebada y trigo (Municipalidad de Sánchez Carrión, 2017).

Por otro lado, el sistema relacional, presenta la dirección de los flujos económicos (productos agropecuarios y sus correspondientes mercados de destinos a nivel local, regional, nacional e internacional), base para la identificación de corredores económicos. Según el Ministerio de Transportes y Comunicaciones (2017), en la provincia de Sánchez Carrión la red vial nacional es de $254.13 \mathrm{~km}$, la departamental es de $51.56 \mathrm{~km}$ y la vecinal de $649.52 \mathrm{~km}$ conectando los centros poblados dispersos, rurales y las ciudades. Cabe resaltar que, de la red vial nacional, el $22.6 \%$ se encuentra asfaltado, $55.7 \%$ afirmado, $11.3 \%$ con pavimento básico, $1.2 \%$ con pavimento rígido y $9.2 \%$ como trocha. Respecto a la red vial departamental, el $99 \%$ se encuentra afirmado, el $0.9 \%$ sin asfaltar y el $0.1 \%$ proyectado, es así que los productos de Sanagorán como el maíz, palta y ganado ovino tienen como principal destino la provincia de Cajabamba (Cajamarca). Por su parte, el eucalipto de Sanagorán se comercializan en Otuzco (Municipalidad de Sánchez Carrión, 2017).

Respecto al Sistema Ambiental, es importante que los planes de desarrollo tengan en consideración la vulnerabilidad de la población (comunidades de personas expuestas) ante riesgos de desastres o peligros, tales como: deslizamientos de rocas y aluviones, inundaciones, minería informal, fallas geológicas, presencia de sequías, zona de alta contaminación, en 
Sanagorán existen 697 familias, siendo el cuarto distrito con $13.95 \%$ que se encuentra expuesta a riesgos de desastres (Municipalidad de Sánchez Carrión, 2017).

La provincia de Sánchez Carrión clasifica a los centros poblados en tres categorías: centro poblado potencial generador de ciudad (mayor a 20000 habitantes), centro poblado rural (entre 151 a 20000 habitantes) y centros poblados dispersos (de 1 a 150 habitantes). En la provincia de Sánchez Carrión, existe solo un centro poblado potencial generador de ciudad (Huamachuco) y se localiza en el distrito de Huamachuco. Por otro parte, se han identificado 209 centros poblados rurales (población entre 151 a 20000 habitantes), de los cuales 44 se encuentran en el distrito de Huamachuco, 38 en Chugay, 23 en Sartimbamba, 22 en Curgos, 19 en Marcabal, 18 en Cochorco, 15 en Sarín y 30 en Sanagorán. Finalmente se registran 324 centros poblados dispersos, de los cuales 77 se localizan en el distrito de Sartimbamba, 62 en Marcabal, 57 en Sarín,31 en Cochorco, 30 en Chuguay, 14 en Curgos, 8 en Huamachuco y por último 45 en Sanagorán (Municipalidad de Sánchez Carrión, 2017).

Finalmente, la relación equipamental en la provincia de Sánchez Carrión está dada por instituciones educativas de nivel escolar, establecimientos de salud y comisarías. En cuanto a las instituciones educativas en la provincia de Sánchez Carrión, existen 852 instituciones educativas; de las cuales 839 pertenece al nivel educativo básico regular, compuesto por 392 instituciones educativas nivel Inicial, 315 nivel primaria y 132 nivel secundaria; así también se consideran 8 instituciones de nivel básica alternativa; 1 institución educativa de nivel básica especial y; por último 4 instituciones educativas de nivel técnico productivo CETPRO. En la provincia de Sánchez Carrión se encuentran 39 establecimientos de salud; en Huamachuco existen 7 establecimientos de salud, de los cuales se considera: 1 hospital de atención general, 1 centro de salud sin internamiento, 2 puestos de salud con médico y 2 puestos de salud sin profesional médico y 1 establecimiento de salud sin categoría; en el distrito de Chugay existen 6 establecimientos de salud de los cuales se considera 1 centro de salud sin internamiento, 2 puestos de salud con médico y 3 puestos de salud sin un profesional médico; en Marcabal se ubican 6 establecimientos de salud, 1 centro de salud con internamiento, 4 puestos de salud con médico y 1 puesto de salud sin profesional médico; en Cochorco existen 5 establecimientos de salud, 4 puestos de salud con médico y 1 puesto de salud sin profesional médico; mientras que en el distrito de Sarín se encuentran 5 establecimientos de salud, 1 centro de salud sin internamiento y 4 puestos de salud con médico; así también en Curgos se ubican 2 establecimientos de salud, 1 centro de salud sin internamiento y 1 puesto de salud sin personal médico; en Sartimbamba consideran 4 establecimientos de salud, 1 centro de salud con internamiento, 2 puestos de salud con profesional médico y 1 puesto de salud sin profesional médico; finalmente, en Sanagorán se ubican 4 centros de salud con profesional médico. Para finalizar, se tiene información de sus autoridades municipales acerca del número de comisarías en la provincia de Sánchez Carrión, concluyéndose que existe 1 comisaría por distrito, excepto en los distritos de Sartimbamba, Cochorco y Sanagorán (Municipalidad de Sánchez Carrión, 2017).

A nivel internacional estudios como el de Alegría (2016) sustentó que el desarrollo económico se logra cuando grupos o comunidades se reúnen en torno a proyectos comunes gestionados eficientemente, basados en unas prácticas estandarizadas (know-how),que aplicados 
en los niveles público, privado a los que denominará "Sistemas de Gestión. Sin embargo, Guevara (2016) quien demuestra que el rol cumple la política fiscal en el crecimiento económico.

Por otro lado, Suanes (2015) refiere que los países con niveles de desigualdad y pobreza más altos tienden a tener un menor crecimiento económico, por sí solo no alcanza para mejorar los resultados de las economías en desarrollo en su proceso de convergencia, por lo tanto, el desafío consiste en encontrar canales efectivos para promover el crecimiento y desarrollo sostenible en el tiempo. La literatura empírica para América Latina se dirige hacia la inversión en actividades de innovación tecnológica, investigación y desarrollo (I + D), como puntos claves para elevar la productividad, explicando así el menor crecimiento económico de la región.

En el contexto peruano autores como Casimiro (2017) expresa que los proyectos de inversión al no tener objetivos o lineamientos de eficiencia durante el ciclo de proyectos se requieren de la experiencia de los evaluadores del proyecto para determinar la correcta relación entre el alcance del proyecto y los aspectos de ingeniería, generalmente en la fase de Pre inversión. Por otro lado, Mendiburu (2016), expresa que se obtuvieron una variación significativa en los proyectos de inversión referidos a los sectores educación y salud. En una muestra de 17 estudios de pre inversión en la región La Libertad, 7 proyectos fueron "Consistente" en un 41\%, 9 estudios fueron "Inconsistente" (regular) del 53\%, mientras que un estudio obtuvo la calificación de "Inconsistente" (débil) en un 6\%. Se concluye que la Propuesta Metodológica para Seguimiento y Evaluación de estudios de pre inversión, se convertirá en un valioso instrumento de control, para la ejecución de presupuestos en los proyectos de inversión pública, dado que permitirá el uso racional y adecuado del presupuesto público para inversiones.

Por otro lado, las entidades del Estado utilizan el presupuesto participativo como instrumento para facilitar las decisiones de inversión y lograr mayor efectividad en sus gestiones. La gran mayoría de objetivos estratégicos y específicos de los Plan de Desarrollo Concertado tanto regional y local, no tienen Proyecto Inversión Pública priorizados en el proceso del presupuesto participativo, observándose un sesgo de las inversiones hacia algunos de los ejes y objetivos estratégicos. Los Proyectos de Inversión Pública, que son elegidos a través del Presupuesto Participativo en los Gobiernos regionales y locales de la Provincia Constitucional del Callao, no se encuentran alineados a los objetivos estratégicos de los Planes de Desarrollo Concertado regionales y locales (Hidalgo y Cornejo, 2017)

La Constitución Política del Perú reconoce el pluralismo económico, garantiza el derecho a la propiedad privada, igualdad entre inversionistas nacionales y extranjeros, la libre iniciativa privada, la libre competencia y la igualdad de trato para todas las actividades económicas.

Es así que la inversión privada tiene mucha participación con respecto a la inversión pública, los últimos tres años ha venido disminuyendo de un 19.62\% (2015) a un 17.25\% (2017), para cerrar con un $17.41 \%$ (información trimestral al 2018). Mientras que la inversión pública durante tres años también ha venido disminuyendo de un $4.99 \%$ (2015) a un $4.51 \%$ (2017), para cerrar con un $3.90 \%$ (información trimestral al 2018). 


\section{Metodología}

La presente investigación se realizó bajo el enfoque cualitativo, los métodos aplicados fueron el estudio de caso, analítico e inductivo, porque se buscó caracterizar y describir aspectos propios de cómo la inversión pública en los sectores educación, saneamiento y transporte ha mejorado la calidad de vida de los pobladores del distrito de Sanagorán porqué ahora los caseríos tienen mejores y eficientes servicios educativos, el sistema de agua potable y alcantarillado ha mejorado cuya finalidad es la de disminuir enfermedades dérmicas gastrointestinales y parasitarias, del mismo modo las carreteras se interconectan para el desarrollo de la zona ganadera, agrícola y comercial. (Kothari, 2004). Asimismo, para la recopilación de datos se aplicó la técnica de entrevista a tres profesionales vinculados a la problemática y el análisis documental de cada proyecto obtenido del Ministerio de Economía y Finanzas. Para el análisis de datos se codificaron las sub categorías apriorísticas, luego con el apoyo del software Atlas.ti se procedió a triangular la data, para finalmente realizar el informe.

Para el desarrollo de la investigación la categoría de estudio fue inversión pública cuyas sub categorías fueron: a) Inversión en educación; b) Inversión en saneamiento; c) Inversión en transporte. En cuanto a la educación, se toma en cuenta la creación y desarrollo evolutivo e histórico de sentido de vida y capacidad de aprovechamiento de todo el trabajo con el que el hombre se esfuerza y al cual se dedica, durante los años de su vida, de manera individual y colectiva; bien bajo su propia administración o bajo la dirección de otros, de organizaciones públicas o privadas (León, 2007). Por saneamiento se entiende como el suministro de instalaciones y servicios que permiten eliminar las aguas residuales. Los sistemas de saneamiento inadecuados constituyen una causa importante de morbilidad en todo el mundo. Se ha probado que la mejora del saneamiento tiene efectos positivos significativos en la salud tanto en el ámbito de los hogares como el de las comunidades. El término saneamiento también hace referencia al mantenimiento de buenas condiciones de higiene gracias a servicios como la recogida de basura y la evacuación de aguas residuales (Organización Mundial Salud 2018). El transporte se refiere al movimiento de un producto de un lugar a otro en su recorrido desde el principio de la cadena de suministro hasta el cliente (Chopra, 2008). Se denomina transporte a un sistema formado por múltiples elementos, siendo tres los fundamentales, la infraestructura, el vehículo y la empresa de servicio que viene a constituir la actividad previamente dicha. Estos elementos están interrelacionados entre sí, pues ninguno es útil sin que los otros existiesen (Cendrero y Truyols, 2008).

\section{Resultados}

Los proyectos de la Municipalidad de Sanagorán declarados como proyectos de inversión viables ascienden a S/.413.80 millones de soles, los proyectos en formulación es de S/.1.55 millones de soles, y los proyectos de inversión aprobados es de S/.3.39 millones de soles, que totalizan un monto de S/.418.75 millones de soles. En la figura 1, se muestran los sectores más representativos son los de Educación con un $21.24 \%$, Saneamiento con un $24.99 \%$ y Transporte por un $27.33 \%$ que sumando los tres sectores ascienden a $73.56 \%$. 


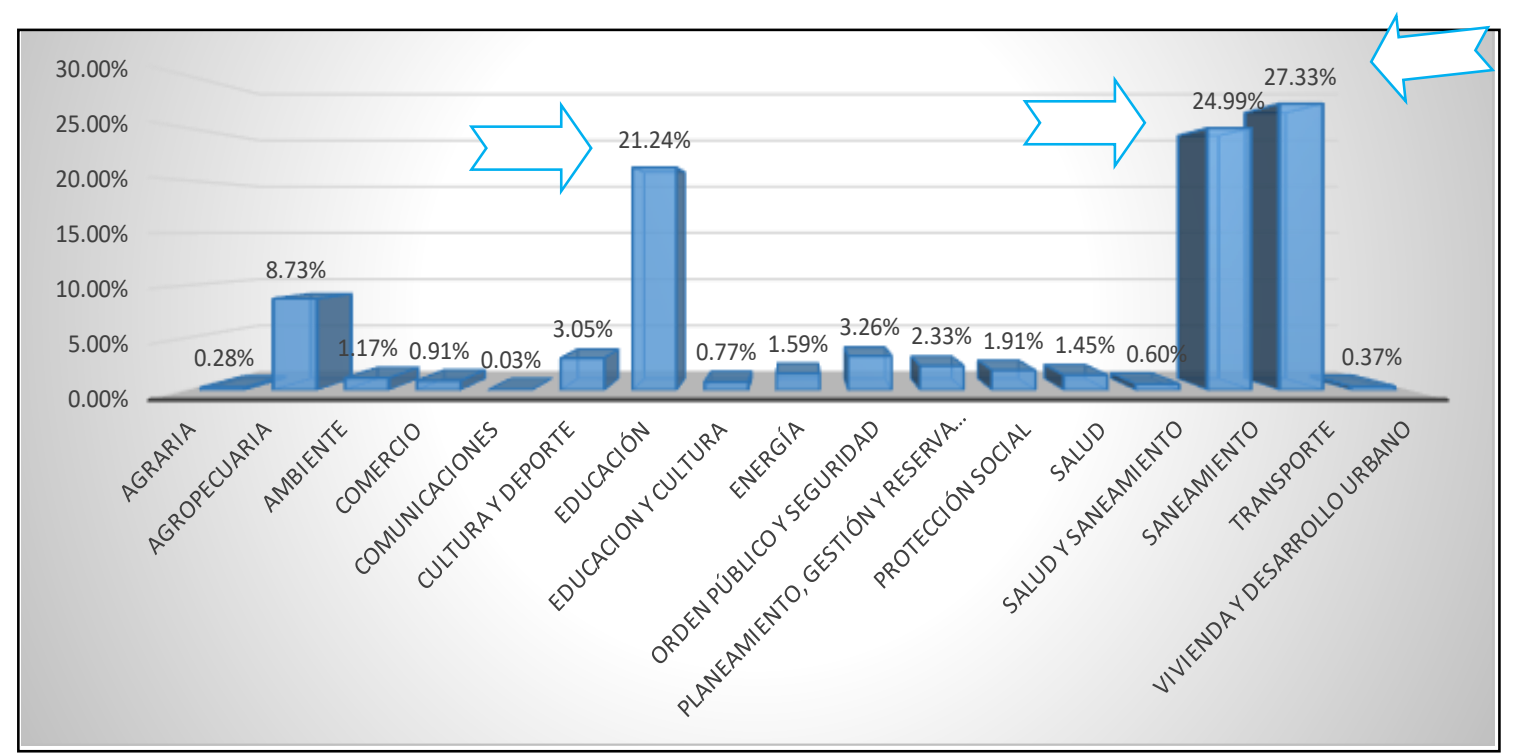

Figura 1 Proyectos de inversión pública por función - Sanagorán 2018

Por ese motivo los proyectos de inversión presentados son aquellos dirigidos a los sectores más vulnerables con la finalidad de elevar la calidad de vida de la población y disminuir así la brecha de necesidades, para el sector educación el nombre del proyecto fue el mejoramiento de los servicios de educación inicial de las instituciones educativas de las diferentes localidades de la provincia de Sánchez Carrión, el cual tiene una zona urbana de $2.15 \%$ y una rural de $97.85 \%$, calculándose un crecimiento poblacional de $1.82 \%$ que incluye los caseríos más alejados como Totoropampa, Pumamullo, Querquerpampa y Malcachugo enfatizando que el 9.44\% deben ser incorporados a la educación básica regular destinándose un total de S/.5,378.232.00 millones de soles a precio de mercado y el precio social es de S/.4,516,314.00, aumentándose así los estándares de calidad de vida logrando que los servicios educativos sean más eficientes dando seguridad física y confort a los beneficiarios mejorando el rendimiento escolar. De acuerdo a las entrevistas realizadas las cuales indican que el nivel actual del estudio del proyecto de inversión pública tiene una modalidad de ejecución de Administración Indirecta, por contrata, siendo el tiempo un indicador útil para la realización del mismo (Ministerio de Economía y Finanzas, 2018).

Para el sector Saneamiento, el proyecto de mayor relevancia fue el de mejoramiento y Ampliación del Sistema de Agua Potable y Saneamiento en la localidad de Sanagorán y del Caserío de Huayobamba, distrito de Sanagorán, provincia de Sánchez Carrión, departamento de La Libertad cuyo objetivo fue disminuir los casos de enfermedades gastrointestinales, parasitarias y dérmicas, en la población de Sanagorán y en el Caserío de Huayobamba, en este último, la población estuvo conformada por 111 familias, con un promedio de 5 habitantes cada una, totalizando 555 personas, además se tiene 7 instituciones públicas que representan el mismo número de familias ascendiendo a 118, lamentablemente esto pobladores consumen agua de deficiente calidad por el deterioro de la infraestructura del sistema de agua, existe poca cobertura, también no cuentan con unidades básicas de saneamiento, ocasionando que la disposición de excretas y las aguas residuales en su mayoría, se vierten hacia los cauces más cercanos, por ende se contamina el flujo y el medio ambiente, peor aún se presentan casos con enfermedades cutáneas y gastrointestinales; en resumen no se cuenta con una cobertura de agua y saneamiento al 
$100 \%$. Por ese motivo el autor del proyecto que está aprobado es el consocio JC, señala que el tiempo también cumple un rol significativo, la modalidad de contrato es por administración indirecta. Por otro lado, las respuestas de los entrevistados consideran que el distrito de Sanagorán tiene pobreza extrema y estos proyectos beneficiarán con servicios básicos y ayudará a que disminuya los riesgos de contraer diversas enfermedades estomacales, ocasionadas por los parásitos y otras enfermedades de la piel, el tener agua potable ayudará la población que mejore su calidad de vida. El monto de la inversión destinado a precio de mercado fue de S/.5.858.773,00 y a precio social por S/.4.604.003,00 (Ministerio de Economía y Finanzas, 2018).

Por último en el sector Transporte se realizó el proyecto de inversión denominado construcción de Trocha Carrozable Huayobamba - sector San Martin, distrito de Sanagorán, provincia de Sánchez Carrión, departamento de La Libertad, cuya finalidad fue implementar el desarrollo agrícola, ganadero y comercial, incrementar la comunicación y sobre todo la movilidad de los pobladores de un lugar a otro, no existía una trocha carrozable que ofreciera conectar Sanagorán y el caserío de Huayobamba en un tramo de $5.57 \mathrm{~km}$. Cabe precisar que el camino vecinal actualmente se encuentra en mal estado debido a derrumbes por la inestabilidad de los taludes, la falta de ensanche en tramos de roca fija, carencia en el mantenimiento anual por parte de la población involucrada. El problema central es el inadecuado nivel de transitabilidad que facilite el traslado de carga y pasajeros entre los caseríos, además que la inexistencia de una trocha carrozable trae consigo pérdidas en sus cosechas. Los principales parámetros y supuestos para la estimación de los beneficios sociales es mejorar los niveles de vida con un adecuado transporte y transitabilidad a los mercados, no obstante, se genera la alteración temporal del suelo, aire y ruido por parte de los obreros durante la construcción.

Uno de los entrevistados manifestó que los tres sectores tienen la misma particularidad para la ejecución de los proyectos, que están relacionados con el uso de los fondos del Estado, los mismos que pueden ser verificados en los reportes de ejecución. En este sentido, todos estos proyectos siguen un proceso en el Project Management Office (PMO) donde se controlan y monitorean las actividades planeadas y ejecutadas.

\section{Conclusiones}

De acuerdo a los resultados obtenidos en la investigación los Proyectos de Inversión Pública están dirigidos básicamente a las necesidades de la población de los sectores, Educación, Saneamiento y Transporte, dado que son los más representativos y tienen la misma particularidad en lo que se refiere a la ejecución de los proyectos, están relacionados con los fondos del Estado y se visualizan en los reportes de ejecución. Tiene un proyecto viable de S/.413.8 Millones soles, los que están en formulación por S/.1.5 millones y en proyectos aprobados por S/.3.39 millones, que totalizan S/.418.75 millones de soles (según MEF), de los cuales los más resaltantes son el Sector Educación con 21.24\%, Saneamiento con $24.99 \%$ y Transporte con $27.33 \%$.

De acuerdo a la entrevista, la ejecución del Proyecto de inversión, cuyo nombre es Mejoramiento de los Servicios de Educación Inicial de las Instituciones Educativas en las localidades de Malcachugo, Totoropampa, Pumamullo y Querquerpampa, distrito de Sanagorán es muy importante para la educación, esto permitirá que la zona rural del distrito pueda acceder a la educación básica aumentando sus estándares de calidad de vida. 
De acuerdo al proyecto sobre mejoramiento y ampliación del Sistema de Agua Potable y Saneamiento en la Localidad de Sanagorán y del Caserío de Huayobamba, Distrito de Sanagorán es importante para la población, dado que ayudará a disminuir los riesgos de contraer enfermedades estomacales, los parásitos y otras enfermedades de la piel, el tener agua potable ayudará a la población a que mejore su calidad de vida.

De acuerdo al proyecto de construcción de Trocha Carrozable Huayobamba, Sector San Martín, Distrito de Sanagorán, los proyectos de inversión en Transporte también son muy importante, porque construir carreteras incrementa la comunicación, el flujo de recursos y sobre todo la movilidad de personas y en particular a los agricultores ayudarán a mejorar la distribución de la producción, de comprar y vender en otros mercados y satisfacer sus necesidades socioeconómicas.

\section{Bibliografía}

Andía, W (2016). Manual de gestión pública (6ta. ed). Lima, Perú: Ediciones Arte \& Pluma.

Andía, W (2010).Gerencia de proyectos sociales y de inversión social. Lima, Perú. Ediciones El saber.

Andía, W (2014).Manual de proyectos de inversión para el sector público. (3era.E.) Lima, Perú. Ediciones Arte \& Pluma.

Anaya, J (2015). El Transporte de mercancías: Enfoque logístico de la distribución: ESIC Editorial, Pozuelo de Alarcón.

Banco Central de Reserva del Perú (2018).Inversión Pública. Recuperado de https:// http://www.bcrp.gob.pe/

Cendrero Agenjo, B. y Truyols Mateu, S (2008). El Transporte: aspectos y tipología, Editorial: Delta Publicaciones.

Collazos, J (2016).Manual de proyectos de inversión privada y pública. (2da.ed.).Lima. Perú: Editorial San Marcos.

Drucker, P (2013). Teorías de la Administración: Administración por objetivos. (2da.ed.). Medellín, Colombia: Editorial Universidad de Medellín.

Escobar, H., Gutiérrez, E. y León, A (2007). Hacienda Pública: Un enfoque económico. (2da.ed.). Medellín, Colombia: Editorial Universidad de Medellín.

Furtado, C (2006). Teoría y Política del Desarrollo Económico. (16ava.E.). México, México. Siglo XXI editores.

Kafka, F (2009).Evaluación Estratégica de Proyectos de Inversión. (2da.ed.) Lima, Perú. Editorial Universidad del Pacífico.

León, A (2007). ¿Qué es la educación?. Caracas, Venezuela. Universidad de los Andes Venezuela.

Ministerio de Economía y Finanzas (2018). Inversión Pública. Recuperado de https://www.mef.gob.pe/es/inversion-publica-sp-21787

Ministerio de Economía y Finanzas (2018). Programa Multianual de inversiones. Recuperado de https://www.mef.gob.pe/es/aplicativos-invierte-pe?id=5634

Morales, A., y Morales, J (2009). Proyectos de Inversión. México D.F. México: Mcgraw-Hill Interamérica.

Organización Mundial de la Salud (2018). Saneamiento. Recuperado de https://www.who.int/topics/sanitation/es/

Esta obra se comparte bajo la licencia Creative Common Atribución-No Comercial 4.0 International (CC BY-NC 4.0)

Revista de la Universidad Internacional del Ecuador. URL: https://www.uide.edu.ec/ 
Pimenta, C. y Pessoa, M (2015).Gestión Financiera Pública en América Latina: La Clave de la Eficiencia y la Transparencia. New York, EE.UU. Banco Interamericano de Desarrollo.

Soto, C y Audar, J (2015). El Sistema Nacional de Inversión Pública (SNIP) y la Preparación de Proyectos de Inversión Pública (PIP).(4ta.Edición). Lima, Perú: Growth Corporation

Torres, J (2013). Teoría General de la Administración: Teoría de la Burocracia. (2 ${ }^{\mathrm{da}}$.Edición). México. Grupo Editorial Patria S.A. de C.V.

Valencia, H., Ponce, A. y Mujica, E (1999). Gobiernos locales y desarrollo rural sostenible en los andes. Lima, Perú: DSGL: Desarrollo Sostenible y Gestión Local.

Vásquez, E (2013).Gestión de la inversión social en América Latina. Lima, Perú. Pearson Educación.

Vera, L (2015). Factores que contribuyeron a prolongar la duración del proceso de formulación de los estudios de pre inversión (perfil, pre - factibilidad y factibilidad) del proyecto de inversión pública denominado "mejoramiento de la atención de las personas con discapacidad de alta complejidad en el instituto nacional de rehabilitación. (Disertación Tesis maestría). Lima: Pontificia Universidad Católica del Perú. Recuperada en: http://tesis.pucp.edu.pe/repositorio/handle/123456789/5908

Villanueva, $\mathrm{P}$ (2013). Inversión privada, inversión pública, términos de intercambio $y$ fluctuaciones en la economía peruana: 1992:1 - 2007. (4ta.Edición).Lima, Perú. Pontificia Universidad Católica del Perú, Facultad de Ciencias Sociales. 